\title{
Autonomous Wireless Sensor Node with Thermal Energy Harvesting for Temperature Monitoring of Industrial Devices
}

\author{
https://doi.org/10.3991/ijoe.v13i04.6802 \\ Liqun Hou \\ North China Electric Power University, Baoding, China \\ houliqunencepu.edu.cn \\ Shudong Tan \\ North China Electric Power University, Baoding, China \\ Lei Yang \\ North China Electric Power University, Baoding, China \\ Zhijuan Zhang \\ North China Electric Power University, Baoding, China \\ Neil W. Bergmann \\ The University of Queensland, Brisbane, Australia
}

\begin{abstract}
The feasibility of using thermal energy harvesting to power a wireless sensor node for temperature monitoring of industrial devices is explored and evaluated in this paper. The thermal energy harvesting equipment and energy conversion circuit have been designed and fabricated. A series of experiments with various sleep periods for the sensor node are undertaken. The experimental results show that the thermal energy harvesting equipment and energy conversion circuit are able to power a commercial wireless node when the sleep period of the node is more than $16 \mathrm{~s}$, equating to a duty cycle of $5.4 \%$. The results also indicate that the wireless sensor network based on the proposed autonomous wireless sensor node can monitor the industrial device temperature successfully.
\end{abstract}

Keywords-wireless sensor networks, energy harvesting, thermoelectric generator, temperature monitoring

\section{$1 \quad$ Introduction}

Harvesting energy from ambient energy sources such as light [1], mechanical vibration [2], and thermal gradient [3] is a promising alternative to batteries for a sustainable wireless sensor network (WSN) node. There are often many hot walls or pipelines at industrial plants even when there is insufficient light or vibration for en- 
ergy harvesting, which motivates this work to examine the feasibility of using thermal gradients for energy harvesting.

Recently, several researchers have explored the use of thermoelectric energy harvesting technology for autonomous sensors or wireless sensor nodes. In [3], a commercial thermoelectric generator (TEG), a TMG 127-1.0-2.5 from Kryotherm, has been successfully used to power an autonomous sensing node for temperature measurement. However, instead of using a commercial wireless sensor node, a simple low power conditioning circuit and RF transmitter are used to interface with a temperature sensor and periodically transmit the measurement information via an RF link. This solution sacrifices the convenience and processing power of a commercial sensor node in exchange for energy saving. A thermoelectric power generation device based on solar irradiation heating, and using a commercial TEG, GM-200-127-14-16 from European Thermodynamics, is described in [4]. The experimental results show that one TEG module is able to generate $4.7 \mathrm{~mW}$ power for wireless sensor nodes or other low power applications at $3.75 \times 104$ lux illumination in full daylight, but the proposed generator has not been proven to actually power a sensor node. A self-powered thermal energy harvesting wireless sensor node for temperature measurement with a small, compact, and mechanically rigid structure is reported in [5]. However, this system uses a custom designed TEG and sensor node, rather than a standard commercial sensor node. In this paper, a complete system is built which shows that a commercial TEG can power a commercial wireless sensor node with a useful duty cycle.

\section{The principle of thermal energy harvesting}

A commercial TEG is generally comprised of many thermocouples connected in series, shown as Fig.1. When there is a temperature difference across a TEG, it will generate electric energy in the level of microwatts/K due to the Seebeck effect. The open circuit voltage of the TEG, $V_{G}$, is proportional to the temperature difference across the TEG according to the following equation [3]:

$$
V_{G}=N \alpha_{p n} \Delta T_{T E G}
$$

Where $N$ is the number of thermocouples, $\alpha_{p n}$ is the Seebeck coefficient of the thermocouple, and $\Delta T_{T E G}$ is the temperature difference across the TEG. $\alpha_{p n}$ and $\triangle T_{T E G}$ are defined as below:

$$
\begin{gathered}
\alpha_{p n}=\alpha_{p}-\alpha_{n} \\
\Delta T_{T E G}=\frac{K}{K+2 K_{i n}} \Delta T=\beta \Delta T
\end{gathered}
$$

where $\alpha_{p}$ and $\alpha_{n}$ are the Seebeck coefficient of p-type and n-type semiconductor, respectively; $\Delta T$ is the temperature difference between the two ends of thermoelectric module, $K$ is the thermal conductivity of the ceramic plate, while $K_{\text {in }}$ is the internal thermal conductivity of the thermocouples. 


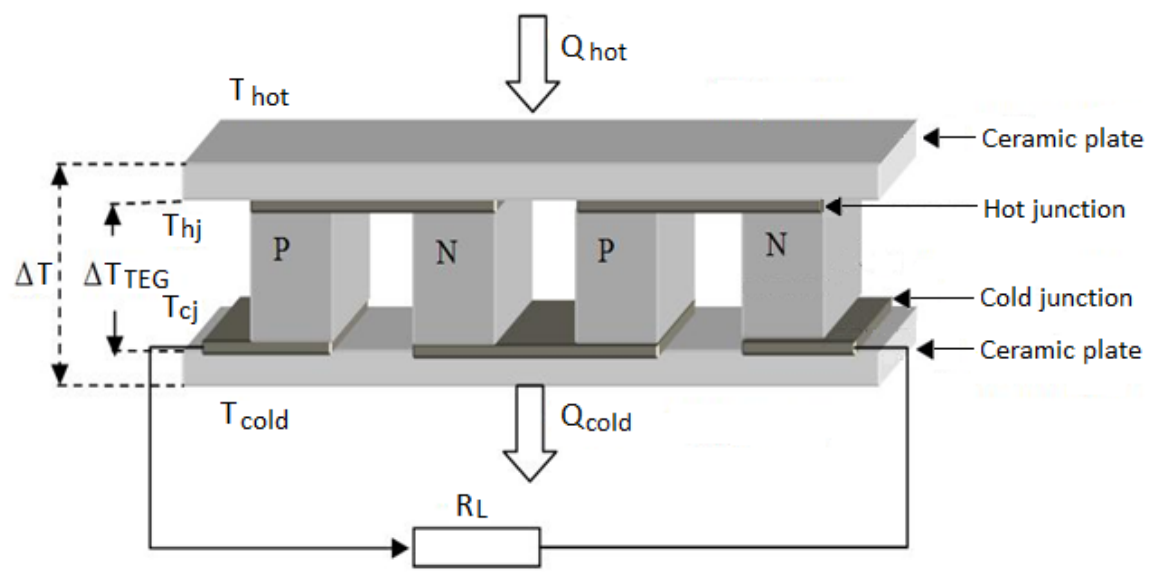

Fig. 1. Structure of TEG elements

\section{System architecture and implementation}

The schematic diagram of the TEG-powered sensor node for industrial temperature monitoring is shown in Fig. 2.

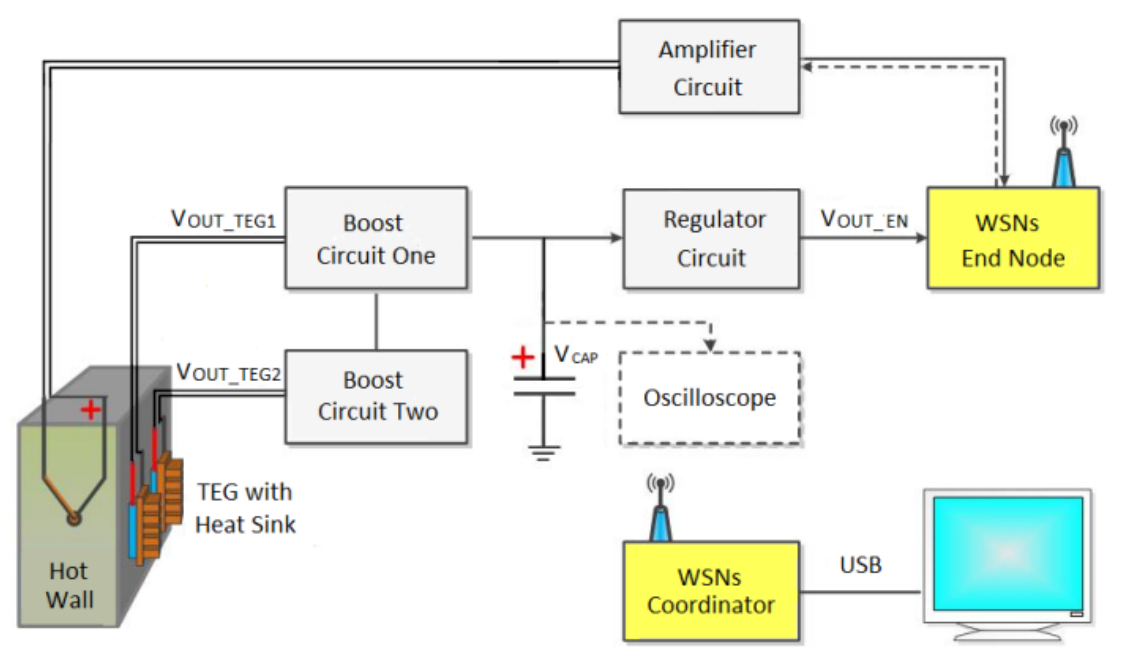

Fig. 2. Schematic diagram of the autonomous WSNs node with thermal energy harvesting for temperature monitoring of industrial devices

According to our previous research [6], the energy harvested by one commercial TEG unit, a TGM287-1.0-1.3 from Kryotherm, with a $\Delta T_{T E G}$ below $15^{\circ} \mathrm{C}$ is insufficient to power a commercial wireless sensor node, so two TGM287-1.0-1.3 modules are used in this design. As shown in Fig. 2, the two TEGs convert thermal energy from the hot wall into electric energy. The output voltage of the two TEGs, VOUT_TEGI 
and $V_{\text {OUT_TEG2 }}$, are boosted by a step-up converter and power manager IC, LTC3108 from Linear Technology. The two LTC3108 ICs are connected in series to charge the supercapacitor. The turns ratio of the small external step-up transformer for the LTC3108 is 1:20. A 4 farad supercapacitor stores the harvested energy. The voltage of the supercapacitor, $V_{C A P}$, is then regulated using a linear regulator, a TPS76801 from TI, to power the sensor node.

A K-type thermocouple is employed in this system to measure the temperature of an industrial device, i.e. the hot wall in this application. The output signal of the thermocouple is amplified by a MC33202 from ON Semiconductor and sampled by a 12bit A/D converter integrated in the Jennic JN5139 wireless sensor node, a typical commercial WSN nodes supporting the ZigBee standard. To save energy, the amplifier is able to synchronously switch between sleep and active modes. The JN5139 controls the amplifier sleep/active through a LP2985 regulator from Texas Instruments. Another two K-type thermocouples and a potentiometer are used in the experimental setup to obtain the hot and cold side temperatures and the temperature difference of the TEG.

A thermoelectric collector, shown as Fig.3, is designed to obtain high temperature difference between the two ends of thermoelectric module and more thernal energy from the hot wall. The collector consists of four thermal conductive solicone pads and two copper plates for heat-transferring from the hot wall to the TEG and then to the copper heatsink, one aerogel blanket for preventing heat-transfer from the heat wall to the TEG, and a cooper heatsink to obain low cold side temperatures of the TEG.

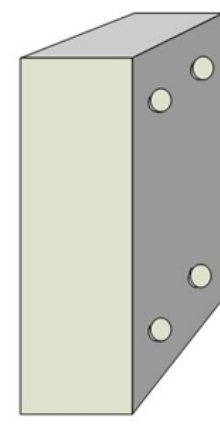

Hot Wall

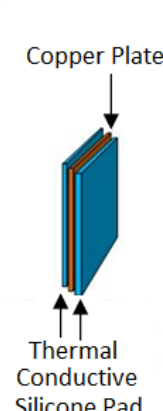

Aerogel Blanket

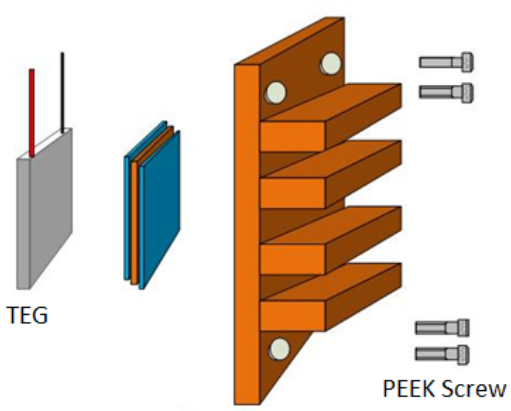

Cooper Heatsink

Fig. 3. Assembly diagram of the thermoelectric collector

\section{Experimental validation}

\subsection{Feasibility of self-power}

There are three mains factors that determine whether a commercial WSN node with thermal energy harvesting can operate autonomously. First is how much energy can be harvested by the TEG, which depends on the temperature difference between 
the hot and cold sides of the TEG; second is how efficiently the energy can be stored and regulated to the required operating voltage of the circuitry, and third is how much energy will be consumed by the wireless sensor node. When the average harvested power exceeds the conversion losses and average sensor node power, the system can operate autonomously, i.e., with an indefinite lifetime.

A series of experiments were carried out using the experimental setup shown in Fig.4. When the hot wall temperature and environmental temperature are set at $67^{\circ} \mathrm{C}$ and $19^{\circ} \mathrm{C}$, which are typical values in an industrial setting, the experimental results indicate that the cold side temperature of the TEG with the specially designed copper heat sink stabilizes at $53^{\circ} \mathrm{C}$. The temperature difference between the two sides of the TEG is $14^{\circ} \mathrm{C}$

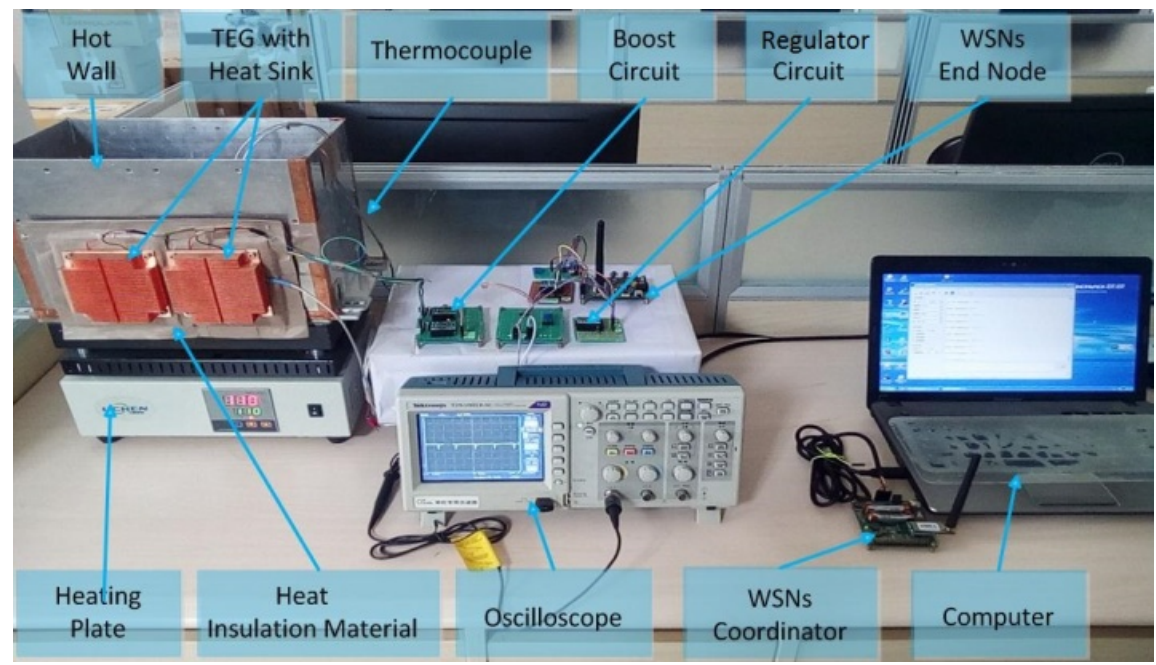

Fig. 4. View of experimental setup

Considering the large difference in energy consumption between the active mode and sleep mode of sensor nodes, duty cycled operation is used in most WSN applications. In this experiment, various sleep periods of the node are tested to see whether the harvested energy under the above-mentioned thermal gradient is sufficient for autonomous operation. The initial voltage value of the super-capacitor $\left(V_{C A P}\right)$ is set at 3.0V. The results in Fig. 5 show that when the sleep periods of the node and the amplifier are $2 \mathrm{~s}, 5 \mathrm{~s}$, and $10 \mathrm{~s}$, the harvested energy is less than the consumed energy, and the supercapacitor discharges and $V_{C A P}$ drops to $2.7 \mathrm{~V}$, the minimum working value of the regulator, in around $10 \mathrm{mins}$, 20mins, and $45 \mathrm{mins}$. With sleep periods of $20 \mathrm{~s}, 30 \mathrm{~s}$, and $60 \mathrm{~s}$, the harvested energy is more than the consumed energy, and the supercapacitor charges, and $V_{C A P}$ climbs to $3.3 \mathrm{~V}$, the maximum voltage value of supercapacitor, in about $75 \mathrm{mins}$, 30mins, and $25 \mathrm{mins}$. When the sleep period is $16 \mathrm{~s}$, the harvested energy is equal to the consumed energy, and $V_{C A P}$ remains fairly constant at $3.0 \mathrm{~V}$. 


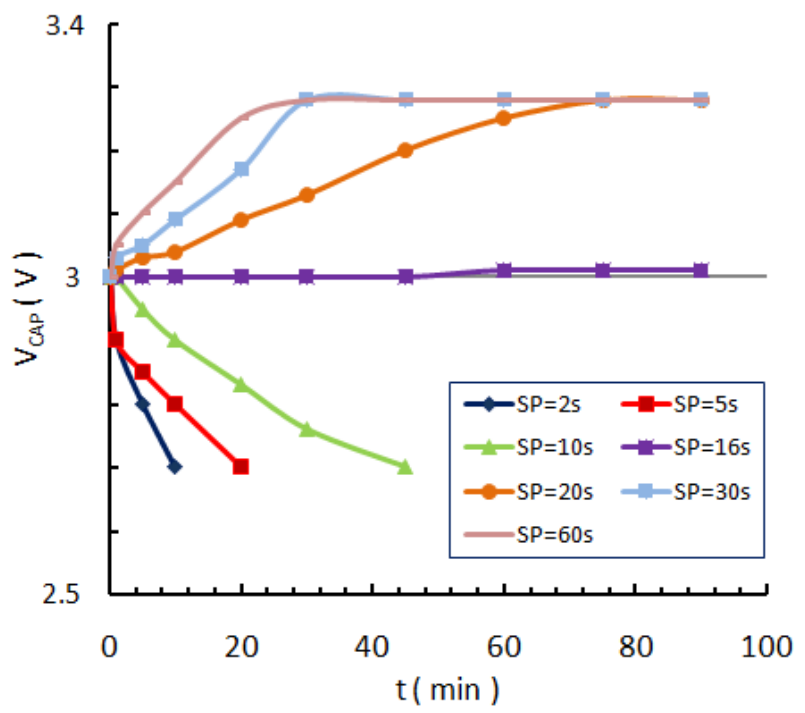

Fig. 5. The charging behaviour of $\mathrm{V}_{\mathrm{CAP}}$ under various sleep periods (SPs)

Fig. 6 shows the detailed waveforms of $V_{C A P}$ at this balance point. The initial waveform of $V_{C A P}$ at time 14:34 is recorded as Fig. 6a, while the waveform of $V_{C A P}$ after 25 minutes is given in Fig. 6b. It is clear that the two waveforms are basically identical.

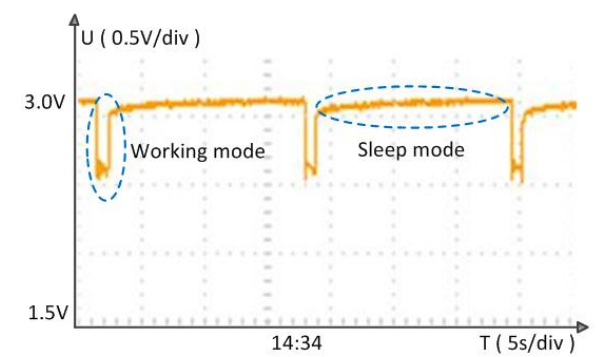

(a)

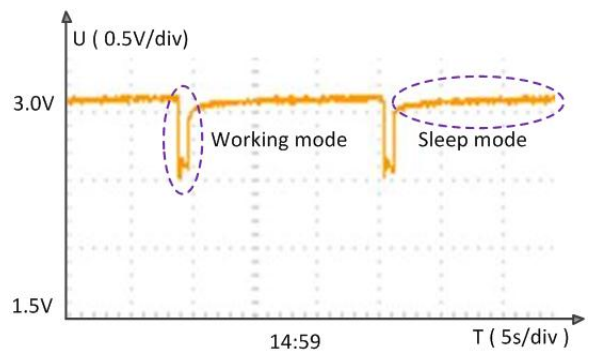

(b)

Fig. 6. The voltage value of the super-capacitor, $V_{C A P}$ $\begin{array}{lll}\text { (a) Initial value (b) Final value after } 25 \text { minutes } & \end{array}$

The energy conversion efficiency of the boost circuit is also measured. The output voltage and current of the boost circuit are $3.28 \mathrm{~V}$ and $1.1 \mathrm{~mA}$, while the input voltage and current of the two boost circuits are $386 \mathrm{mV}, 17 \mathrm{~mA}$, and $389 \mathrm{mV}, 17 \mathrm{~mA}$. The output power of the boost circuit is $3.6 \mathrm{~mW}$, while the input power is $13.1 \mathrm{~mW}$ ( $6.5 \mathrm{~mW}$ for TEG one and 6.6 for TEG two). So the energy coversion rate of the boost circuit is about $27 \%$. 


\subsection{Temperature monitoring}

In the experimental setup shown as Fig.4, the hot wall temperature and environmental temperature are monitored by the wireless sensor network composed of the proposed autonomous wireless sensor node. The experimental curves are recorded as Fig.7. In the graph, the two curves represent the hot wall temperature and environmental temperature respectively. The experimental result indicates that the proposed wireless sensor node can work autonomously and complete temperature signal monitoring successfully.

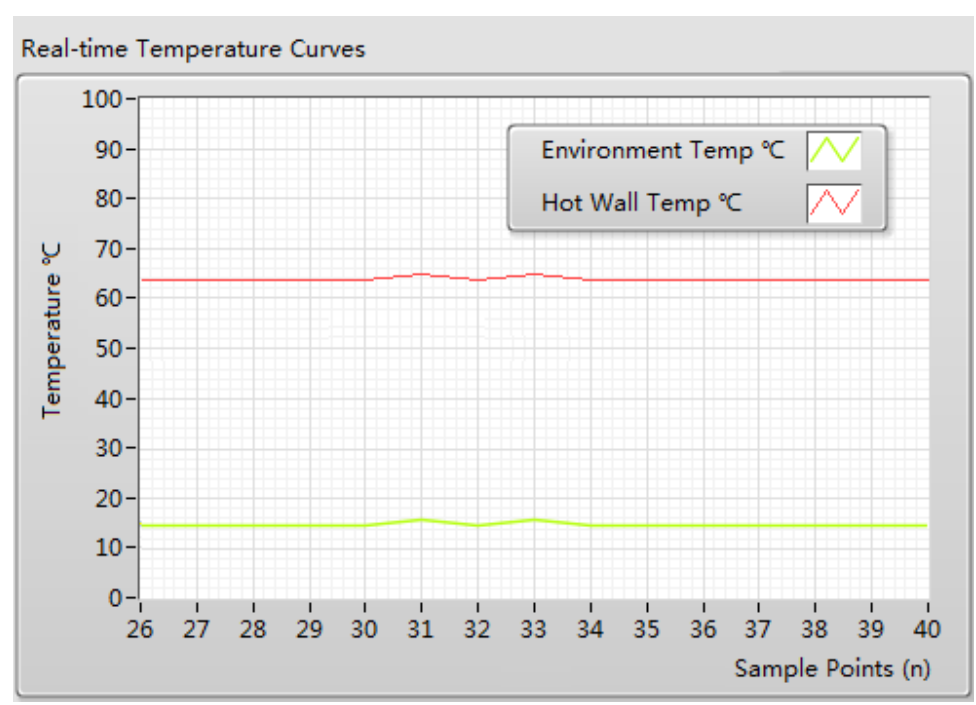

Fig. 7. The recorded temperature curves

\section{Conclusion}

The feasibility of using thermal energy harvesting to power a commercial wireless sensor node for temperature monitoring of industrial equipment has been demonstrated. The experimental results show that the energy harvested by the thermal energy harvesting equipment and energy conversion circuit is able to power a commercial wireless node with an active period of $0.9 \mathrm{~s}$ when the sleep period of the node is more than 16 s, i.e., when the duty cycle is around $5.4 \%$. The results also indicate that the wireless sensor network based on the proposed autonomous wireless sensor node can monitor the industrial device temperature successfully.

\section{Acknowledgment}

This work was supported by the Scientific Research Foundation for the Returned Overseas Chinese Scholars, The Ministry of Education of the People's Republic of 
China, the Natural Science Foundation of Hebei Province, China (Grant No. F2016502104), and the Fundamental Research Funds for the Central Universities of China.

\section{$7 \quad$ References}

[1] López-Lapeña, O., Penella, M. T., and Gasulla, M.: 'A closed-loop maximum power point tracker for subwatt photovoltaic panels', 2012, IEEE Transactions on Industrial Electronics, 59(3), pp.1588-1596 https://doi.org/10.1109/TIE.2011.2161254

[2] Dayal, R., Dwari, S., and Parsa, L.: 'Maximum energy harvesting from vibration-based electromagnetic microgenerator using active damping' , 2010, Electronics Letters, 46(5), pp.371 - 373 https://doi.org/10.1049/el.2010.3264

[3] Dalola, S., Ferrari, M., Ferrari, V., Guizzetti, M., Marioli, D., and Taroni, A.: 'Characterization of thermoelectric modules for powering autonomous sensors', IEEE Transactions on Instrumentation and Measurement, 2009, 58(1), pp.99-107 https://doi.org/10.1109/TIM. 2008.928405

[4] Zhang, Z., Li, W., and Kan, J.: 'Behavior of a thermoelectric power generation device based on solar irradiation and the earth's surface-air temperature difference', 2015, Energy Conversion and Management, 97, pp.178-187 https://doi.org/10.1016/j.enconman.2015. $\underline{03.060}$

[5] Prijić, A., Vračar, L., Vučković, D., Milić D., Prijić, Z.: 'Thermal energy harvesting wireless sensor node in aluminum core PCB technology', 2015, IEEE Sensors Journal, 15(1), pp.337-345 https://doi.org/10.1109/JSEN.2014.2343932

[6] Hou, L., Tan, S.: 'A preliminary study of thermal energy harvesting for industrial wireless sensor networks', 10th International Conference on Sensing Technology (ICST), Nanjing, China, Nov. 2016, pp. 386-390. https://doi.org/10.1109/icsenst.2016.7796283

\section{Authors}

Liqun Hou (corresponding author) is an Associate Professor in the School of Control and Computer Engineering, North China Electric Power University, Baoding 071003, China. E-mail: houliqun@ncepu.edu.cn.

Shudong Tan is with the School of Control and Computer Engineering, North China Electric Power University, Baoding 071003, China. E-mail: tsd132@163.com.

Lei Yang is with the School of Control and Computer Engineering, North China Electric Power University, Baoding 071003, China. E-mail: yl_126_mail@126.com.

Zhijuan Zhang is an Associate Professor in the School of Electrical and Electronic Engineering, North China Electric Power University, Baoding 071003, China. Email: zhzhj@ncepu.edu.cn.

Neil W. Bergmann is a Professor of Embedded Systems in the School of Information Technology and Electrical Engineering, The University of Queensland, Brisbane, Qld. 4072. E-mail: bergmann@itee.uq.edu.au.

Article submitted 24 February 2017. Published as resubmitted by the authors 15 April 2017. 